\title{
A unified method for boundedness in fully parabolic chemotaxis systems with signal-dependent sensitivity
}

\author{
Masaaki Mizukami \\ Department of Mathematics, Tokyo University of Science \\ 1-3, Kagurazaka, Shinjuku-ku, Tokyo 162-8601, Japan \\ masaaki.mizukami.math@gmail.com \\ Tomomi Yokota* $\dagger$ \\ Department of Mathematics, Tokyo University of Science \\ 1-3, Kagurazaka, Shinjuku-ku, Tokyo 162-8601, Japan \\ yokota@rs.kagu.tus.ac.jp
}

September 17, 2018

\begin{abstract}
This paper deals with the Keller-Segel system

$$
\begin{cases}u_{t}=\Delta u-\nabla \cdot(u \chi(v) \nabla v), & x \in \Omega, t>0, \\ v_{t}=\Delta v+u-v, & x \in \Omega, t>0,\end{cases}
$$

where $\Omega$ is a bounded domain in $\mathbb{R}^{n}$ with smooth boundary $\partial \Omega, n \geq 2 ; \chi$ is a function satisfying $\chi(s) \leq K(a+s)^{-k}$ for some $k \geq 1$ and $a \geq 0$. In the case that $k=1$, Fujie (J. Math. Anal. Appl.; 2015; 424; 675-684) established global existence of bounded solutions under the condition $K<\sqrt{\frac{2}{n}}$. On the other hand, when $k>1$, Winkler (Math. Nachr.; 2010; 283; 1664-1673) asserted global existence of bounded solutions for arbitrary $K>0$. However, there is a gap in the proof. Recently, Fujie tried modifying the proof; nevertheless it also has a gap. It seems to be difficult to show global existence of bounded solutions for arbitrary $K>0$. Moreover, the condition for $K$ when $k>1$ cannot connect to the condition when $k=1$. The purpose of the present paper is to obtain global existence and boundedness under more natural and proper condition for $\chi$ and to build a mathematical bridge between the cases $k=1$ and $k>1$.
\end{abstract}

2010Mathematics Subject Classification. Primary: 35K51; Secondary: 35B45, 35A01, 92C17.

Key words and phrases: chemotaxis; sensitivity function; global existence; boundedness.

* Corresponding author

${ }^{\dagger}$ Partially supported by Grant-in-Aid for Scientific Research (C), No. 16K05182. 


\section{Introduction}

The chemotaxis system proposed by Keller and Segel in [9] describes a part of the life cycle of cellular slime molds with chemotaxis. After the pioneering work [9], a number of variations of the chemotaxis system are proposed and investigated (see e.g., $[\mathbf{1}, \mathbf{6}, \mathbf{7}]$ ).

In this paper we consider the Keller-Segel system with signal-dependent sensitivity

$$
\begin{cases}u_{t}=\Delta u-\nabla \cdot(u \chi(v) \nabla v), & x \in \Omega, t>0, \\ v_{t}=\Delta v+u-v, & x \in \Omega, t>0, \\ \nabla u \cdot \nu=\nabla v \cdot \nu=0, & x \in \partial \Omega, t>0, \\ u(x, 0)=u_{0}(x), v(x, 0)=v_{0}(x), & x \in \Omega,\end{cases}
$$

where $\Omega$ is a bounded domain in $\mathbb{R}^{n}(n \geq 2)$ with smooth boundary $\partial \Omega$ and $\nu$ is the outward normal vector to $\partial \Omega$. The initial data $u_{0}$ and $v_{0}$ are assumed to be nonnegative functions. The unknown function $u(x, t)$ represents the population density of species and $v(x, t)$ shows the concentration of the substance at place $x$ and time $t$. As to the sensitivity function $\chi$, we are interested in functions generalizing

$$
\chi(s)=\frac{K}{s} \quad \text { and } \quad \chi(s)=\frac{K}{(1+s)^{2}} \quad(s>0) .
$$

In a mathematical view, the difficulty caused by the sensitivity function is to deal with the additional term $u \chi^{\prime}(v)|\nabla v|^{2}$ which does not appear in the case that $\chi$ is a constant. Moreover, when $\chi$ is the singular sensitivity function, it is delicate to derive the estimate for $\frac{1}{v}$. In the case that $\chi(v)=\frac{K}{v}$, Winkler [14] first attained global existence of classical solutions when $K<\sqrt{\frac{2}{n}}$ and global existence of weak solutions when $K<\sqrt{\frac{n+2}{3 n-4}}$. However, the result in $[\mathbf{1 4}]$ could not arrive at boundedness of solutions to (1.1). To overcome the difficulty in a singular sensitivity, Fujie [2] established the uniform-in-time lower estimate for $v$, and show global existence of classical bounded solutions to (1.1) in the case that $\chi(v)=\frac{K}{v}$ with

$$
K<\sqrt{\frac{2}{n}}
$$

As to the problem (1.1) with $\chi(v)=\frac{K}{v}$ in the 2-dimensional setting, Lankeit [10] obtained global existence of classical bounded solutions when $K<\chi_{0}\left(\chi_{0}>1\right)$. Futhermore FujieSenba [4] dealt with the 2-dimensional problem which was replaced $v_{t}$ with $\tau v_{t}$ in (1.1) and showed global existence and boundedness of radially symmetric solutions under the condition that $\chi(s) \rightarrow 0(t \rightarrow \infty)$ and $\tau$ is sufficiently small. On the other hand, in the case that $\chi(v) \leq \frac{K}{(1+\alpha v)^{k}}(k>1, \alpha>0, K>0)$, Winkler [13] established global existence and boundedness in (1.1) without any restriction on $K>0$. The result in [13] affected the result in [5] which mentioned global existence of classical bounded solutions to (1.1) when $\chi$ is the strong singular sensitivity function such that $\chi(v)=\frac{K}{v^{k}}$ for $k>1$, and the method in [13] was used in Zhang-Li [15] and Zheng-Mu [16]. However, we cannot convince the result in [13] that global existence and boundedness hold for all $K>0$, because there is a gap in the proof. Recently, Fujie tried modifying it; nevertheless it also 
has a gap (cf. [3]). In general, it seems to be difficult to show global existence of bounded solutions for arbitrary $K>0$. Moreover, if $k \rightarrow 1$, then the condition "arbitrary $K>0$ " cannot connect to (1.2).

The purpose of this paper is to obtain global existence and boundedness in (1.1) under a more natural and proper condition for $\chi$ and to build a mathematical bridge between the cases $k=1$ and $k>1$. We shall suppose that $\chi$ satisfies that

$$
\chi(s) \leq \frac{K}{(a+s)^{k}}
$$

with some $k \geq 1, a \geq 0$ and $K>0$ fulfiling

$$
K<k(a+\eta)^{k-1} \sqrt{\frac{2}{n}}
$$

Here

$$
\eta:=\sup _{\tau>0}\left(\min \left\{e^{-2 \tau} \min _{x \in \bar{\Omega}} v_{0}(x), c_{0}\left\|u_{0}\right\|_{L^{1}(\Omega)}\left(1-e^{-\tau}\right)\right\}\right),
$$

where $c_{0}>0$ is a lower bound for the fundamental solution of $w_{t}=\Delta w-w$ with Neumann boundary condition (for more detail, see Remark 2.1). We suppose that

$$
0 \leq u_{0} \in C(\bar{\Omega}) \backslash\{0\} \quad \text { and } \quad \begin{cases}0<v_{0} \in W^{1, q}(\Omega)(\exists q>n) & (a=0) \\ 0 \leq v_{0} \in W^{1 . q}(\Omega) \backslash\{0\}(\exists q>n) & (a>0)\end{cases}
$$

Now the main results read as follows.

Theorem 1.1. Let $n \geq 2$ and let $\Omega \subset \mathbb{R}^{n}$ be a bounded domain with smooth boundary. Assume that $\chi$ satisfies (1.3) with some $k \geq 1, a \geq 0, K>0$ fulfiling (1.4). Then for any $u_{0}, v_{0}$ satisfying (1.6) with some $q>n$, there exists an exactly one pair $(u, v)$ of functions

$$
u, v \in C(\bar{\Omega} \times[0, \infty)) \cap C^{2,1}(\bar{\Omega} \times(0, \infty))
$$

which solves (1.1). Moreover, the solution $(u, v)$ is uniformly bounded, i.e., there exists a constant $C>0$ such that

$$
\|u(\cdot, t)\|_{L^{\infty}(\Omega)}+\|v(\cdot, t)\|_{L^{\infty}(\Omega)} \leq C \quad \text { for all } t \geq 0 .
$$

Remark 1.1. The unified condition (1.3) with $K>0$ satisfying (1.4) may be a natural condition for $\chi$. Indeed, when $k=1,(1.4)$ becomes (1.2):

$$
K<\left.\left(k(a+\eta)^{k-1} \sqrt{\frac{2}{n}}\right)\right|_{k=1}=\sqrt{\frac{2}{n}} .
$$

The main theorem tells us the result in the typical case of singular sensitivity.

Corollary 1.2. Let $\chi(s)=\frac{K}{s}$ with $K<\sqrt{\frac{2}{n}}$. Then for any $u_{0}, v_{0}$ satisfying (1.6) with some $q>n$, (1.1) has a unique global bounded classical solution. 
The strategy for the proof of Theorem 1.1 is to construct the estimate for $\int_{\Omega} u^{p}$ with some $p>\frac{n}{2}$. One of the keys for this strategy is to derive the unified inequality

$$
\frac{d}{d t} \int_{\Omega} u^{p} \varphi(v)+\varepsilon p(p-1) \int_{\Omega} u^{p-2} \varphi(v)|\nabla u|^{2} \leq c \int_{\Omega} u^{p} \varphi(v)-r \int_{\Omega} u^{p+1} \frac{\varphi(v)}{(a+v)^{k}}
$$

for some $\varepsilon \in[0,1)$ and $c>0$, where

$$
\varphi(s):=\exp \left\{-r \int_{0}^{s} \frac{1}{(a+s)^{k}} d \tau\right\}
$$

with $r>0$. This function $\varphi$ constructed in $[\mathbf{1 1}, \mathbf{1 2}]$ unifies mathematical structures in the cases $k>1$ and $k=1$.

This paper is organized as follows. In Section 2 we collect basic facts which will be used later. In Section 3 we give a unified view point in energy estimates. Section 4 is devoted to the proof of global existence and boundedness (Theorem 1.1).

\section{Preliminaries}

In this section we will collect elementary results. We first recall the uniform-in-time lower estimate for $v$ established by Fujie $[2,3]$.

Lemma 2.1. Let $u_{0} \in C(\bar{\Omega})$ and $u \in C(\bar{\Omega} \times[0, T))$ be nonnegative functions such that $u_{0} \not \equiv 0$ and $\int_{\Omega} u(\cdot, t)=\int_{\Omega} u_{0}(t \in[0, T))$. If $v_{0} \in C(\bar{\Omega})$ is a positive function in $\bar{\Omega}$ and $v \in C^{2,1}(\bar{\Omega} \times(0, T)) \cap C(\bar{\Omega} \times[0, T))$ is a classical solution of

$$
\begin{cases}v_{t}=\Delta v-v+u, & x \in \Omega, t \in(0, T) \\ \nabla v \cdot \nu=0 & x \in \partial \Omega, t \in(0, T), \\ v(x, 0)=v_{0}(x) & x \in \Omega,\end{cases}
$$

then for all $t \in(0, T)$,

$$
\inf _{x \in \Omega} v(x, t) \geq \eta>0
$$

where $\eta>0$ is defined as (1.5).

Remark 2.1. When $\Omega$ is a convex bounded domain, the proof of this lemma is given in $[\mathbf{2}$, Lemma 2.2] and the constant $\eta$ can be explicitly represented as

$$
\eta:=\sup _{\tau>0}\left(\min \left\{e^{-\tau} \min _{x \in \bar{\Omega}} v_{0}(x),\left\|u_{0}\right\|_{L^{1}(\Omega)} \cdot \int_{0}^{\tau} \frac{1}{(4 \pi r)^{\frac{n}{2}}} e^{-\left(r+\frac{\left(\max _{x, y \in \bar{\Omega}}|x-y|\right)^{2}}{4 r}\right)} d r\right\}\right) .
$$

On the other hand, if we do not assume the convexity of $\Omega$, then using the positivity of the fundamental solution $U(t, x ; s, y)$ to $w_{t}=\Delta w-w$ in $\Omega \times(0, T)$ with $\nabla w \cdot \nu=0$ on $\partial \Omega \times(0, T)$ (see e.g., [8]), we have that there exists $c_{0}>0$ such that for all $\tau \in\left(0, \frac{T}{2}\right)$,

$$
U(s+\tau, x ; s, y)=U(\tau, x ; 0, y) \geq c_{0}>0 \quad \text { for all } x, y \in \bar{\Omega}, s>0 .
$$

Then we can see the conclusion of Lemma 2.1 by a similar argument as in [2, Lemma 2.2]. 
We next recall the well-known result about local existence of solutions to (1.1) (see [10, Theorem 2.3], [4, Proposition 2.2] and [13, Lemma 2.1]).

Lemma 2.2. Assume that $\chi$ satisfies (1.3) and the initial data $u_{0}, v_{0}$ fulfil (1.6) for some $q>n$. Then there exist $T_{\max } \in(0, \infty]$ and exactly one pair $(u, v)$ of nonnegative functions

$$
\begin{aligned}
& u \in C\left(\bar{\Omega} \times\left[0, T_{\max }\right)\right) \cap C^{2,1}\left(\bar{\Omega} \times\left(0, T_{\max }\right)\right), \\
& v \in C\left(\bar{\Omega} \times\left[0, T_{\max }\right)\right) \cap C^{2,1}\left(\bar{\Omega} \times\left(0, T_{\max }\right)\right) \cap L_{\mathrm{loc}}^{\infty}\left(\left[0, T_{\max }\right) ; W^{1, q}(\Omega)\right)
\end{aligned}
$$

which solves (1.1) in the classical sense and satisfies the mass conservation

$$
\int_{\Omega} u(\cdot, t)=\int_{\Omega} u_{0} \quad \text { for all } t \in\left(0, T_{\max }\right)
$$

and the lower estimate

$$
\inf _{x \in \Omega} v(x, t) \geq \eta \quad \text { for all } t \in\left(0, T_{\max }\right),
$$

where $\eta>0$ is defined as (1.5). Moreover, if $T_{\max }<\infty$, then

$$
\lim _{t \succ T_{\max }}\left(\|u(\cdot, t)\|_{L^{\infty}(\Omega)}+\|v(\cdot, t)\|_{W^{1, \infty}(\Omega)}\right)=\infty .
$$

At the end of this section we shall recall the result about the estimate for $v$ in dependence on boundedness features of $u$ derived by a straightforward application of well-known smoothing estimates for the heat semigroup under homogeneous Neumann boundary conditions (see [14, Lemma 2.4] and [2, Lemma 2.4]).

Lemma 2.3. Let $T>0$ and $1 \leq \theta, \mu \leq \infty$. If $\frac{n}{2}\left(\frac{1}{\theta}-\frac{1}{\mu}\right)<1$, then there exists $C_{1}(\mu, \theta)>0$ such that

$$
\|v(\cdot, t)\|_{L^{\mu}(\Omega)} \leq C_{1}(\mu, \theta)\left(1+\sup _{s \in(0, \infty)}\|u(\cdot, s)\|_{L^{\theta}(\Omega)}\right) \quad \text { for all } t \in(0, T) .
$$

\section{A unified view point in energy estimates}

Let $(u, v)$ be the solution of $(1.1)$ on $\left[0, T_{\max }\right)$ as in Lemma 2.2. For the proof of Theorem 1.1 we will recall an useful fact to derive the $L^{\infty}$-estimate for $u$.

Lemma 3.1. Assume that the solution u of (1.1) given in Lemma 2.2 satisfies

$$
\|u(\cdot, t)\|_{L^{p}(\Omega)} \leq C(p) \quad \text { for all } t \in\left(0, T_{\max }\right)
$$

with some $p>\frac{n}{2}$ and $C(p)>0$. Then there exists a constant $C^{\prime}>0$ such that

$$
\|u(\cdot, t)\|_{L^{\infty}(\Omega)} \leq C^{\prime} \quad \text { for all } t \in\left(0, T_{\max }\right) .
$$

Proof. Combination of (2.2) and the same argument as in [14, Lemma 3.4] leads to the conclusion of Lemma 3.1. 
Unified test function. Thanks to Lemmas 2.2 and 3.1 we will only make sure that the $L^{p}$-estimate for $u$ with some $p>\frac{n}{2}$ to show global existence and boundedness of solutions to (1.1). To establish (3.1) we introduce the functions $g$ and $\varphi$ by

$$
g(s):=-r \int_{\eta}^{s} \frac{1}{(a+\tau)^{k}} d \tau, \quad \varphi(s):=\exp \{g(s)\} \quad(s \geq \eta),
$$

where $r>0$ is a constant fixed later and $\eta$ is defined as (1.5). When $k>1$, by straightforward calculations we have

$$
\varphi(s)=C_{\varphi} \exp \left\{\frac{r}{(k-1)(a+s)^{k-1}}\right\}
$$

with $C_{\varphi}=\exp \left\{-r(k-1)^{-1}(a+\eta)^{-k+1}\right\}>0$, which is a similar function used in [13]. On the other hand, when $k=1$, it follows that

$$
\varphi(s)=\frac{C_{\varphi}}{(a+s)^{r}}
$$

with some constant $C_{\varphi}=(a+\eta)^{r}>0$, which is a similar function used in [2]. Now we shall prove the following unified inequality by using the test function $\varphi(v)$.

Lemma 3.2. Assume that $\chi$ satisfies (1.3). Then for all $\varepsilon \in[0,1)$ there exists $c>0$ such that

$$
\begin{aligned}
\frac{d}{d t} \int_{\Omega} u^{p} \varphi(v) & +\varepsilon p(p-1) \int_{\Omega} u^{p-2} \varphi(v)|\nabla u|^{2} \\
& \leq \int_{\Omega} u^{p} H_{\varepsilon}(v) \varphi(v)|\nabla v|^{2}+c \int_{\Omega} u^{p} \varphi(v)-r \int_{\Omega} u^{p+1} \frac{\varphi(v)}{(a+v)^{k}}
\end{aligned}
$$

where

$$
H_{\varepsilon}(s):=\frac{(\varepsilon p+1-\varepsilon) r^{2}}{(1-\varepsilon)(p-1)(a+s)^{2 k}}+\frac{\varepsilon p K r}{(1-\varepsilon)(a+s)^{2 k}}-\frac{k r}{(a+s)^{k+1}}+\frac{p(p-1) K^{2}}{4(1-\varepsilon)(a+s)^{2 k}} .
$$

Proof. We let $p \geq 1$. From (1.1) we have

$$
\frac{d}{d t} \int_{\Omega} u^{p} \varphi(v)=p \int_{\Omega} u^{p-1} \varphi(v) \nabla \cdot(\nabla u-u \chi(v) \nabla v)+\int_{\Omega} u^{p} \varphi^{\prime}(v)(\Delta v-v+u) .
$$

Integration by parts yields

$$
\begin{aligned}
& p \int_{\Omega} u^{p-1} \varphi(v) \nabla \cdot(\nabla u-u \chi(v) \nabla v)+\int_{\Omega} u^{p} \varphi^{\prime}(v) \Delta v \\
& =-p \int_{\Omega} \nabla\left(u^{p-1} \varphi(v)\right) \cdot(\nabla u-u \chi(v) \nabla v)-\int_{\Omega} \nabla\left(u^{p} \varphi^{\prime}(v)\right) \cdot \nabla v \\
& =-p(p-1) \int_{\Omega} u^{p-2} \varphi(v)|\nabla u|^{2}+\int_{\Omega} u^{p-1}\left(p(p-1) \varphi(v) \chi(v)-2 p \varphi^{\prime}(v)\right) \nabla u \cdot \nabla v \\
& \quad+\int_{\Omega} u^{p}\left(-\varphi^{\prime \prime}(v)+p \varphi^{\prime}(v) \chi(v)\right)|\nabla v|^{2} .
\end{aligned}
$$


Invoking to the Young inequality, we infer that for all $\varepsilon \in[0,1)$,

$$
\begin{aligned}
& \int_{\Omega} u^{p-1}\left(p(p-1) \varphi(v) \chi(v)-2 p \varphi^{\prime}(v)\right) \nabla u \cdot \nabla v \\
& \leq(1-\varepsilon) p(p-1) \int_{\Omega} u^{p-2} \varphi(v)|\nabla u|^{2}+\int_{\Omega} u^{p} \frac{\left(p(p-1) \varphi(v) \chi(v)-2 p \varphi^{\prime}(v)\right)^{2}}{4(1-\varepsilon) p(p-1) \varphi(v)}|\nabla v|^{2} .
\end{aligned}
$$

Thus combination of (3.4), (3.5) and (3.6) yields that

$$
\frac{d}{d t} \int_{\Omega} u^{p} \varphi(v)+\varepsilon p(p-1) \int_{\Omega} u^{p-2} \varphi(v)|\nabla u|^{2} \leq \int_{\Omega} u^{p} F_{\varphi}(v)|\nabla v|^{2}+\int_{\Omega} u^{p} \varphi^{\prime}(v)(-v+u),
$$

where

$$
F_{\varphi}(s):=-\varphi^{\prime \prime}(s)-\frac{\varepsilon}{1-\varepsilon} p \varphi^{\prime}(s) \chi(s)+\frac{p(p-1)}{4(1-\varepsilon)} \chi(s)^{2} \varphi(s)+\frac{p \varphi^{\prime}(s)^{2}}{(1-\varepsilon)(p-1) \varphi(s)} .
$$

Noting that

$$
\varphi^{\prime}(s)=g^{\prime}(s) \varphi(s) \quad \text { and } \quad \varphi^{\prime \prime}(s)=g^{\prime \prime}(s) \varphi(s)+g^{\prime}(s)^{2} \varphi(s),
$$

we can rewrite the function $F_{\varphi}(s)$ as

$$
F_{\varphi}(s)=\left(-g^{\prime \prime}(s)-\frac{\varepsilon}{1-\varepsilon} p g^{\prime}(s) \chi(s)+\frac{p(p-1)}{4(1-\varepsilon)} \chi(s)^{2}+\frac{(\varepsilon p+1-\varepsilon) g^{\prime}(s)^{2}}{(1-\varepsilon)(p-1)}\right) \varphi(s) .
$$

Recalling by (3.2) that

$$
g^{\prime}(s)=\frac{-r}{(a+s)^{k}} \quad \text { and } \quad g^{\prime \prime}(s)=\frac{r k}{(a+s)^{k+1}},
$$

we see from (1.3) that

$$
F_{\varphi}(s) \leq H_{\varepsilon}(s) \varphi(v)
$$

where

$$
H_{\varepsilon}(s):=\frac{(\varepsilon p+1-\varepsilon) r^{2}}{(1-\varepsilon)(p-1)(a+s)^{2 k}}+\frac{\varepsilon p K r}{(1-\varepsilon)(a+s)^{2 k}}-\frac{k r}{(a+s)^{k+1}}+\frac{p(p-1) K^{2}}{4(1-\varepsilon)(a+s)^{2 k}} .
$$

Therefore we obtain from (3.7) together with (3.8) that

$$
\begin{aligned}
\frac{d}{d t} \int_{\Omega} u^{p} \varphi(v) & +\varepsilon p(p-1) \int_{\Omega} u^{p-2} \varphi(v)|\nabla u|^{2} \\
& \leq \int_{\Omega} u^{p} H_{\varepsilon}(v) \varphi(v)|\nabla v|^{2}-r \int_{\Omega} u^{p} \varphi(v) \frac{(-v+u)}{(a+v)^{k}} .
\end{aligned}
$$

We finally confirm from the boundedness of $\frac{s}{(a+s)^{k}}(k \geq 1)$ that there exists $c>0$ satisfying

$$
\int_{\Omega} u^{p} \varphi(v) \frac{r v}{(a+v)^{k}} \leq c \int_{\Omega} u^{p} \varphi(v)
$$

and thus we obtain (3.3). 


\section{Global existence and boundedness}

In this section we will show the $L^{p}$-estimate for $u$ with $p>\frac{n}{2}$ by using Lemma 3.2.

\subsection{Energy estimate in the case $k>1$}

We first derive the energy estimate in the case $k>1$. In this subsection we assume that $\chi$ satisfies (1.3) with some $k>1$. Now we shall show the following inequality by modifying the method in [11].

Lemma 4.1. Assume that (1.3) and (1.4) are satisfied with some $k>1, a \geq 0$ and $K>0$. Then there exist $p>\frac{n}{2}, r>0$ and $\varepsilon \in(0,1)$ such that

$$
H_{\varepsilon}(s) \leq 0 \quad \text { for all } s \geq \eta,
$$

where $H_{\varepsilon}$ is defined in Lemma 3.2 and $\eta>0$ is defined as (1.5), which implies that

$$
\frac{d}{d t} \int_{\Omega} u^{p} \varphi(v)+\varepsilon p(p-1) \int_{\Omega} u^{p-2} \varphi(v)|\nabla u|^{2} \leq c \int_{\Omega} u^{p} \varphi(v)-r \int_{\Omega} u^{p+1} \frac{\varphi(v)}{(a+v)^{k}} .
$$

Proof. We take $p \geq 1, r>0$ and $\varepsilon \in[0,1)$ which will be fixed later. Due to the definition of $H_{\varepsilon}$ we write as

$$
H_{\varepsilon}(s)=a_{1}(s) r^{2}+a_{2}(s) r+a_{3}(s) \text {, }
$$

where

$$
\begin{aligned}
& a_{1}(s):=\frac{\varepsilon p+1-\varepsilon}{(1-\varepsilon)(p-1)(a+s)^{2 k}}, \\
& a_{2}(s):=\frac{\varepsilon p K}{(1-\varepsilon)(a+s)^{2 k}}-\frac{k}{(a+s)^{k+1}}, \\
& a_{3}(s):=\frac{p(p-1) K^{2}}{4(1-\varepsilon)(a+s)^{2 k}} .
\end{aligned}
$$

Noting from the condition (1.4) that there exist $p>\frac{n}{2}$ and $\varepsilon \in(0,1)$ satisfying

$$
K<\frac{(1-\varepsilon) k(a+\eta)^{k-1}}{\varepsilon p+\sqrt{p(\varepsilon p+1-\varepsilon)}}
$$

we have that

$$
\frac{(\varepsilon p+\sqrt{p(\varepsilon p+1-\varepsilon)})}{1-\varepsilon} K \leq k(a+s)^{k-1} \text { for all } s \geq \eta
$$

This implies that the discriminant

$$
\begin{aligned}
D_{r}(s) & =a_{2}(s)^{2}-4 a_{1}(s) a_{3}(s) \\
& =\left(\frac{\varepsilon p K}{(1-\varepsilon)(a+s)^{2 k}}-\frac{k}{(a+s)^{k+1}}\right)^{2}-\frac{p(\varepsilon p+1-\varepsilon) K^{2}}{(1-\varepsilon)^{2}(a+s)^{4 k}} \\
& =\frac{1}{(a+s)^{4 k}}\left(\left(\frac{\varepsilon p K}{(1-\varepsilon)}-k(a+s)^{k-1}\right)^{2}-\frac{p(\varepsilon p+1-\varepsilon) K^{2}}{(1-\varepsilon)^{2}}\right)
\end{aligned}
$$


is nonnegative for all $s \geq \eta$. Finally, we show that there exists $r>0$ such that

$$
H_{\varepsilon}(s)=a_{1}(s) r^{2}+a_{2}(s) r+a_{3}(s) \leq 0
$$

for all $s \geq \eta$. Because $D_{r}(s)$ is nonnegative, we can define

$$
\begin{aligned}
r_{ \pm}(s) & :=\frac{-a_{2} \pm \sqrt{D_{r}(s)}}{2 a_{1}(s)} \\
& =\frac{(1-\varepsilon)(p-1)}{2(\varepsilon p+1-\varepsilon)}\left(-\frac{\varepsilon p K}{(1-\varepsilon)}+k(a+s)^{k-1} \pm(a+s)^{2 k} \sqrt{D_{r}(s)}\right) .
\end{aligned}
$$

Then we see that $H_{\varepsilon}(s) \leq 0$ for each $s \geq \eta$ and all $r \in\left(r_{-}(s), r_{+}(s)\right)$. Since the functions

$$
\widetilde{r}_{ \pm}(\tau):=\tau \pm \sqrt{\tau^{2}-\frac{p(\varepsilon p+1-\varepsilon) K^{2}}{(1-\varepsilon)^{2}}} \quad\left(\tau \geq \frac{K \sqrt{p(\varepsilon p+1-\varepsilon)}}{1-\varepsilon}\right)
$$

satisfy that

$$
\frac{d \widetilde{r}_{+}}{d \tau}(\tau)>0 \quad \text { and } \quad \frac{d \widetilde{r}_{-}}{d \tau}(\tau)<0
$$

for all $\tau \geq \frac{K \sqrt{p(\varepsilon p+1-\varepsilon)}}{1-\varepsilon}$, we obtain that

$$
r_{-}(s) \leq \widetilde{r}_{-}\left(\frac{K \sqrt{p(\varepsilon p+1-\varepsilon)}}{1-\varepsilon}\right)=\widetilde{r}_{+}\left(\frac{K \sqrt{p(\varepsilon p+1-\varepsilon)}}{1-\varepsilon}\right) \leq r_{+}(s)
$$

holds for all $s \geq \eta$. Therefore if we put

$$
r_{0}:=\widetilde{r}_{-}\left(\frac{K \sqrt{p(\varepsilon p+1-\varepsilon)}}{1-\varepsilon}\right)=\frac{(p-1) K^{2}}{2} \sqrt{\frac{p}{\varepsilon p+1-\varepsilon}}>0,
$$

then $r=r_{0} \in\left(r_{-}(s), r_{+}(s)\right)$ for all $s \geq \eta$, which means that (4.2) holds for all $s \geq \eta$. This implies the end of the proof.

Now we are ready to show the $L^{p}$-estimate in the case $k>1$.

Lemma 4.2. Assume that (1.3) and (1.4) are satisfied with some $k>1, a \geq 0$ and $K>0$. Then there exist $p>\frac{n}{2}$ and $C>0$ such that

$$
\|u(\cdot, t)\|_{L^{p}(\Omega)} \leq C \quad \text { for all } t>0 .
$$

Proof. The proof is similar as in [13]. From Lemmas 3.2 and 4.1 we obtain (4.1) with some $p>\frac{n}{2}, r>0$ and $\varepsilon \in(0,1)$. We shall show the $L^{p}$-estimate for $u$ by using (4.1). From the positivity of $u, v$ and $\varphi$ we have that

$$
-r \int_{\Omega} u^{p+1} \frac{\varphi(v)}{(a+v)^{k}} \leq 0 .
$$


We next deal with the term $\int_{\Omega} u^{p-1} \varphi(v)|\nabla u|^{2}$. Noting the boundedness of $\varphi$ :

$$
C_{\varphi} \leq \varphi(s) \leq 1 \quad(s \geq \eta)
$$

and combining the Gagliardo-Nirenberg inequality with the mass conservation property (2.1), we deduce that there exists $c_{1}>0$ such that

$$
\begin{aligned}
\int_{\Omega} u^{p} \varphi(v) & \leq \int_{\Omega} u^{p}=\left\|u^{\frac{p}{2}}\right\|_{L^{2}(\Omega)}^{2} \\
& \leq c_{1}\left(\left\|\nabla u^{\frac{p}{2}}\right\|_{L^{2}(\Omega)}+\left\|u^{\frac{p}{2}}\right\|_{L^{\frac{2}{p}}(\Omega)}\right)^{2 a}\left\|u^{\frac{p}{2}}\right\|_{L^{\frac{2}{p}}(\Omega)}^{2(1-a)} \\
& =c_{1}\left(\left\|\nabla u^{\frac{p}{2}}\right\|_{L^{2}(\Omega)}+\left\|u_{0}\right\|_{L^{1}(\Omega)}^{\frac{p}{2}}\right)^{2 a}\left\|u_{0}\right\|_{L^{1}(\Omega)}^{p(1-a)}
\end{aligned}
$$

with $a=\frac{\frac{2 n}{p}-\frac{n}{2}}{\frac{2 n}{p}+1-\frac{n}{2}} \in(0,1)$. From (4.1), (4.3) and combination of (4.5) and

$$
C_{\varphi} \int_{\Omega}\left|\nabla u^{\frac{p}{2}}\right|^{2} \leq \int_{\Omega} u^{p-2} \varphi(v)|\nabla u|^{2}
$$

we see that there exist $c_{2}, c_{3}>0$ such that

$$
\frac{d}{d t} \int_{\Omega} u^{p} \varphi(v) \leq c \int_{\Omega} u^{p} \varphi(v)-c_{2}\left(\int_{\Omega} u^{p} \varphi(v)\right)^{\frac{1}{a}}+c_{3},
$$

which implies that there exist $p>\frac{n}{2}, r>0$ (determined in Lemma 4.1) and $C>0$ satisfying

$$
\int_{\Omega} u^{p} \varphi(v) \leq C
$$

Therefore we obtain from (4.4) that

$$
\int_{\Omega} u^{p} \leq C C_{\varphi}^{-1}
$$

Thus we attain the goal of the proof.

\subsection{Energy estimates in the case $k=1$}

In this section we assume that the sensitivity function $\chi$ satisfies (1.3) with $k=1$. We first show the following estimate for $H_{0}(s)$.

Lemma 4.3. Assume that (1.3) and (1.4) are satisfied with $k=1$ and with some $a \geq 0$ and $K>0$. Then for all $p \in\left(1, \frac{1}{K^{2}}\right)$ there exists an interval $I_{p}$ such that for all $r \in I_{p}$ and $s \geq \eta$,

$$
H_{0}(s) \leq 0
$$

where $H_{0}$ is defined as in Lemma 3.2 with $\varepsilon=0$, which means

$$
\frac{d}{d t} \int_{\Omega} u^{p} \varphi(v) \leq c \int_{\Omega} u^{p} \varphi(v)-r \int_{\Omega} u^{p+1} \frac{\varphi(v)}{a+v} .
$$


Proof. We pick $p \geq 1$ and $r>0$ which will be fixed later. Due to the definition of $H_{0}(s)$ we write as

$$
\begin{aligned}
H_{0}(s) & =\frac{1}{(a+s)^{2}}\left(\frac{1}{p-1} r^{2}-r+\frac{p(p-1) K^{2}}{4}\right) \\
& =: \frac{b_{1} r^{2}+b_{2} r+b_{3}}{(a+s)^{2}} .
\end{aligned}
$$

From (1.4) we note that $\left(1, \frac{1}{K^{2}}\right)$ is not an empty set. If we choose $p \in\left(1, \frac{1}{K^{2}}\right)$, then the discriminant of $b_{1} r^{2}+b_{2} r+b_{3}$ is nonnegative:

$$
D_{r}=1-p K^{2} \geq 0 .
$$

Thus we can define the interval $I_{p}$ as

$$
I_{p}:=\left(\frac{p-1}{2}\left(1-\sqrt{1-p K^{2}}\right), \frac{p-1}{2}\left(1+\sqrt{1-p K^{2}}\right)\right)
$$

for each $p \in\left(1, \frac{1}{K^{2}}\right)$. Then we have from straightforward calculations that for each $p \in\left(1, \frac{1}{K^{2}}\right)$ and all $r \in I_{p}, b_{1} r^{2}+b_{2} r+b_{3} \leq 0$ holds, which yields that for all $p \in\left(1, \frac{1}{K^{2}}\right)$ there exists the interval $I_{p}$ such that

$$
H_{0}(s) \leq 0
$$

for all $r \in I_{p}$. This implies the end of the proof.

We next show the estimate for $\int_{\Omega} u^{p} \varphi(v)$. However we cannot easily obtain the estimate for $\int_{\Omega} u^{p}$ because $\varphi$ is not a bounded function. Therefore we will show the following lemma, which has an important role for obtaining the $L^{p}$-estimate.

Lemma 4.4. Assume that (1.3) and (1.4) are satisfied with $k=1$ and with some $a \geq 0$ and $K>0$. Suppose that $p \in\left(1, \frac{1}{K^{2}}\right), r \in I_{p}$ such that $p-r \geq 1$. If there exists $C>0$ such that

$$
\|v(\cdot, t)\|_{L^{p-r}(\Omega)} \leq C \quad \text { for all } t>0
$$

then there exists $C_{2}(p, r)>0$ satisfying

$$
\int_{\Omega} u^{p} \varphi(v) \leq C_{2}(p, r) \quad \text { for all } t>0 .
$$

Proof. The proof is similar as in $[2]$. We let $p \in\left(1, \frac{1}{K^{2}}\right)$. We denote by $I_{p}$ the interval defined in Lemma 4.3, and choose $r \in I_{p}$. We shall attain the conclusion from (4.6). By virtue of the Hölder inequality, we infer that

$$
\begin{aligned}
\int_{\Omega} u^{p} \varphi(v) & =C_{\varphi}^{-\frac{p}{p+1}} \int_{\Omega}\left(u^{p+1} \frac{C_{\varphi}}{(a+v)^{r+1}}\right)^{\frac{p}{p+1}} \cdot(a+v)^{-r+\frac{p(r+1)}{p+1}} \\
& \leq C_{\varphi}^{-\frac{p}{p+1}}\left(\int_{\Omega} u^{p+1} \frac{C_{\varphi}}{(a+v)^{r+1}}\right)^{\frac{p}{p+1}}\left(\int_{\Omega}(a+v)^{p-r}\right)^{\frac{1}{p+1}} \\
& =C_{\varphi}^{-\frac{p}{p+1}}\left(\int_{\Omega} u^{p+1} \frac{\varphi(v)}{a+v}\right)^{\frac{p}{p+1}}\left(\int_{\Omega}(a+v)^{p-r}\right)^{\frac{1}{p+1}} .
\end{aligned}
$$


Noting from (4.7) and the fact $p-r \geq 1$ that

$$
\|a+v(\cdot, t)\|_{L^{p-r}(\Omega)} \leq a+C \text { for all } t>0,
$$

we obtain that there exists $c_{1}>0$ such that

$$
\int_{\Omega} u^{p} \varphi(v) \leq c_{1}\left(\int_{\Omega} u^{p+1} \frac{\varphi(v)}{a+v}\right)^{\frac{p}{p+1}} .
$$

Plugging (4.8) into (4.6) yields that there exists $c_{2}>0$ such that

$$
\frac{d}{d t} \int_{\Omega} u^{p} \varphi(v) \leq c \int_{\Omega} u^{p} \varphi(v)-c_{2}\left(\int_{\Omega} u^{p} \varphi(v)\right)^{\frac{p+1}{p}} .
$$

Therefore we have from a standard ODE comparison argument that there exists $C>0$ such that

$$
\int_{\Omega} u^{p} \varphi(v) \leq C
$$

which means the end of the proof.

Lemma 4.5. Assume that (1.3) and (1.4) are satisfied with $k=1$ and with some $a \geq 0$ and $K>0$. Then there exist $p>\frac{n}{2}$ and $C>0$ such that

$$
\|u(\cdot, t)\|_{L^{p}(\Omega)} \leq C .
$$

Proof. We use a similar argument as in [2, Proof of Main Theorem (Step 1)]. First we choose a pair $\left(p_{0}, r_{0}\right)$ such that

$$
\left\{\begin{array}{l}
p_{0} \in\left(1, \min \left\{\frac{1}{K^{2}}, n+1, \frac{n+2}{n-2}\right\}\right), \\
r_{0}:=\frac{p_{0}-1}{2}
\end{array}\right.
$$

Then $p_{0}>r_{0}, r_{0}<\frac{n}{2}, r_{0} \in I_{p}, p_{0}-r_{0} \geq 1$ and $p_{0}-r_{0}<\frac{n}{n-2}$. Since $\frac{n}{2}\left(1-\frac{1}{p_{0}-r_{0}}\right)<1$ from the inequality $p_{0}-r_{0}<\frac{n}{n-2}$, it follows from Lemma 2.3 and (2.1) that there exists $b_{0}>0$ such that

$$
\|v(\cdot, t)\|_{L^{p_{0}-r_{0}(\Omega)}} \leq C_{1}\left(p_{0}-r_{0}, 1\right)\left(1+\sup _{s \in(0, \infty)}\|u(\cdot, s)\|_{L^{1}(\Omega)}\right) \leq b_{0} .
$$

Thus we have from the fact $p_{0}-r_{0} \geq 1$ and Lemma 4.4 that

$$
\int_{\Omega} u^{p_{0}}(a+v)^{-r_{0}} \leq C_{\varphi}^{r_{0}} C_{2}\left(p_{0}, r_{0}\right)
$$

We will show that for all $q_{0} \in\left(\frac{2 p_{0}}{p_{0}+1}, \min \left\{p_{0}, \frac{n\left(p_{0}-r_{0}\right)}{n-2 r_{0}}\right\}\right)$ there exists $b_{0}^{\prime}>0$ such that

$$
\int_{\Omega} u^{q_{0}} \leq b_{0}^{\prime}
$$


We first note from the fact $p_{0} \notin\left(\frac{n}{n+4}, 1\right)$ that

$$
\frac{2 p_{0}}{p_{0}+1} \leq p_{0} \quad \text { and } \quad \frac{2 p_{0}}{p_{0}+1} \leq \frac{n\left(p_{0}+1\right)}{2\left(n-p_{0}+1\right)}=\frac{n\left(p_{0}-r_{0}\right)}{n-2 r_{0}}
$$

Now we fix $q_{0} \in\left(\frac{2 p_{0}}{p_{0}+1}, \min \left\{p_{0}, \frac{n\left(p_{0}-r_{0}\right)}{n-2 r_{0}}\right\}\right)$. Applying the Hölder inequality, we infer that

$$
\begin{aligned}
\int_{\Omega} u^{q_{0}} & \leq\left(\int_{\Omega} u^{p_{0}}(a+v)^{-r_{0}}\right)^{\frac{r_{0}}{p_{0}}} \cdot\left(\int_{\Omega}(a+v)^{\frac{q_{0} r_{0}}{p_{0}-q_{0}}}\right)^{\frac{p_{0}-r_{0}}{p_{0}}} \\
& \leq\left(C_{\varphi}^{r_{0}} C_{2}\left(p_{0}, r_{0}\right)\right)^{\frac{r_{0}}{p_{0}}} \cdot\|a+v(\cdot, t)\|_{L^{\frac{q_{0} r_{0}}{p_{0}}}}^{\frac{q_{0} r_{0}}{p_{0}-r_{0}}(\Omega)} .
\end{aligned}
$$

We can confirm from the fact $\frac{n}{2}\left(\frac{1}{q_{0}}-\frac{p_{0}-q_{0}}{q_{0} r_{0}}\right)<1$ and Lemma 2.3 that

$$
\|v(\cdot, t)\|_{L^{\frac{q_{0} r_{0}}{p^{-}-r_{0}}(\Omega)}} \leq C_{1}\left(\frac{q_{0} r_{0}}{p_{0}-r_{0}}, q_{0}\right)\left(1+\sup _{s \in(0, \infty)}\|u(\cdot, s)\|_{L^{q_{0}}(\Omega)}\right) .
$$

Therefore plugging (4.11) and the inequality $\frac{q_{0} r_{0}}{p_{0}-r_{0}} \geq 1$ (from the fact $q_{0} \geq \frac{2 p_{0}}{p_{0}+1}$ ) into (4.10) derives that there exists $b_{0}^{\prime \prime}>0$ satisfying

$$
\|u(\cdot, t)\|_{L^{q_{0}(\Omega)}} \leq b_{0}^{\prime \prime}\left(1+\sup _{s \in(0, \infty)}\|u(\cdot, s)\|_{L^{q_{0}(\Omega)}}\right)^{\frac{r_{0}}{p_{0}}} .
$$

Noting that $\frac{r_{0}}{p_{0}}<1$, we obtain (4.9). In the above argument, since $\frac{n}{2}<\min \left\{\frac{1}{K^{2}}, n+1\right\}$, if $\frac{n+2}{n-2}>\frac{n}{2}$, then we can fix $p_{0}>\frac{n}{2}$. Thus we can find $q_{0}>\frac{n}{2}$ due to $p_{0}<\frac{n\left(p_{0}+1\right)}{2\left(n-p_{0}+1\right)}=\frac{n\left(p_{0}-r_{0}\right)}{n-2 r_{0}}$ if $\frac{n+2}{n-2}>\frac{n}{2}$. On the other hand, if $\frac{n+2}{n-2} \leq \frac{n}{2}$, then we proceed the iteration argument. For all $i \in \mathbb{N}$, we fix a pair $\left(p_{i}, r_{i}\right)$ defined inductively such as

$$
\left\{\begin{array}{l}
p_{i} \in\left(p_{i-1}, \min \left\{\frac{1}{K^{2}}, n+1, \frac{p_{i-1}(n+2)}{n-2 p_{i-1}}\right\}\right), \\
r_{i}:=\frac{p_{i}-1}{2}
\end{array}\right.
$$

then we can see that $p_{i}>r_{i}, r_{i}<\frac{n}{2}$ and $r_{i} \in I_{p_{i}}$. Moreover, since the relation $p_{i}<\frac{p_{i-1}(n+2)}{n-2 p_{i-1}}$ implies that

$$
p_{i}-r_{i}<\frac{n \cdot \frac{n\left(p_{i-1}+1\right)}{2\left(n-p_{i-1}+1\right)}}{n-2 \cdot \frac{n\left(p_{i-1}+1\right)}{2\left(n-p_{i-1}+1\right)}},
$$

we can find some $q_{i-1} \in\left(\frac{2 p_{i-1}}{p_{i-1}+1}, \min \left\{p_{i-1}, \frac{n\left(p_{i-1}+1\right)}{2\left(n-p_{i-1}+1\right)}\right\}\right)$ satisfying

$$
p_{i}-r_{i}<\frac{n q_{i-1}}{n-2 q_{i-1}}
$$

which means that $\frac{n}{2}\left(\frac{1}{q_{i-1}}-\frac{1}{p_{i}-r_{i}}\right)<1$. Therefore we obtain

$$
\|v(\cdot, t)\|_{L^{p_{i}-r_{i}(\Omega)}} \leq C_{1}\left(p_{i}-r_{i}, q_{i-1}\right)\left(1+\sup _{s \in(0, \infty)}\|u(\cdot, s)\|_{L^{q_{i-1}(\Omega)}}\right) .
$$


According to (4.12) and $p_{i}-r_{i} \geq 1$ into Lemma 4.4, we have

$$
\int_{\Omega} u^{p_{i}}(a+v)^{-r_{i}} \leq C_{\varphi}^{r_{i}} C_{2}\left(p_{i}, r_{i}\right)
$$

Due to a similar argument to the first iteration, we can show that

$$
\|u(\cdot, t)\|_{L^{q_{i}(\Omega)}} \leq b_{i}^{\prime}\left(q_{i}\right) \quad \text { for all } q_{i} \in\left(\frac{2 p_{i}}{p_{i}+1}, \min \left\{p_{i}, \frac{n\left(p_{i}-r_{i}\right)}{n-2 r_{i}}\right\}\right)
$$

with some constant $b_{i}^{\prime}\left(q_{i}\right)>0$. Because the increasing function $f(x):=\frac{x(n+2)}{n-2 x}$ satisfies $f(x)>1$ and $f(x) \rightarrow \infty$ as $x \rightarrow \frac{n}{2}$, we can find some $i_{0} \in \mathbb{N}$ such that $p_{i_{0}}>\frac{n}{2}$ and $p_{i_{0}-1} \leq \frac{n}{2}$, and hence $q_{i_{0}}>\frac{n}{2}$. Therefore we verify

$$
\|u(\cdot, t)\|_{L^{p}(\Omega)} \leq C
$$

with some $p>\frac{n}{2}$ and some $C>0$, which completes the proof.

\subsection{Proof of Theorem 1.1}

Combination of the $L^{p}$-estimate for $u$ (see Lemma 4.2 or Lemma 4.5) and Lemma 3.1 directly leads to Theorem 1.1. 


\section{References}

[1] N. Bellomo, A. Bellouquid, Y. Tao, M. Winkler, Toward a mathematical theory of KellerSegel models of pattern formation in biological tissues, Math. Models Methods Appl. Sci. 25 (2015), 1663-1763.

[2] K. Fujie, Boundedness in a fully parabolic chemotaxis system with singular sensitivity, J. Math. Anal. Appl. 424 (2015), 675-684.

[3] K. Fujie, Study of reaction-diffusion systems modeling chemotaxis, Doctoral thesis, 2016.

[4] K. Fujie, T. Senba, Global existence and boundedness of radial solutions to a two dimensional fully parabolic chemotaxis system with general sensitivity, Nonlinearity, to appear.

[5] K. Fujie, T. Yokota, Boundedness in a fully parabolic chemotaxis system with strongly singular sensitivity, Appl. Math. Lett. 38 (2014), 140-143.

[6] T. Hillen, K. J. Painter, A user's guide to PDE models for chemotaxis, J. Math. Biol. 58 (2009), 183-217.

[7] D. Horstmann, From 1970 until present: the Keller-Segel model in chemotaxis and its consequences, Jahresber. Deutsch. Math. -Verein. 106 (2004), 51-69.

[8] S. Itô, Diffusion equations, Translated from the 1979 Japanese original by the author. Translations of Mathematical Monographs, 114. American Mathematical Society, Providence, RI, 1992.

[9] E. F. Keller, L. A. Segel, Initiation of slime mold aggregation viewed as an instability, J. Theor. Biol. 26 (1970), 399-415.

[10] J. Lankeit, A new approach toward boundedness in a two-dimensional parabolic chemotaxis system with singular sensitivity, Math. Methods Appl. Sci. 39 (2016), 394-404.

[11] M. Mizukami, T. Yokota, Global existence and asymptotic stability of solutions to a twospecies chemotaxis system with any chemical diffusion, J. Differential Equations 261 (2016), 2650-2669.

[12] M. Negreanu, J. I. Tello, On a two species chemotaxis model with slow chemical diffusion, SIAM J. Math. Anal. 46 (2014), 3761-3781.

[13] M. Winkler, Absence of collapse in a parabolic chemotaxis system with signal-dependent sensitivity, Math. Nachr. 283 (2010), 1664-1673.

[14] M. Winkler, Global solutions in a fully parabolic chemotaxis system with singular sensitivity, Math. Methods Appl. Sci. 34 (2011), 176-190.

[15] Q. Zhang, Y. Li, Global boundedness of solutions to a two-species chemotaxis system, Z. Angew. Math. Phys. 66 (2015), 83-93.

[16] P. Zheng, C. Mu, Global existence of solutions for a fully parabolic chemotaxis system with consumption of chemoattractant and logistic source, Math. Nachr. 288 (2015), 710-720. 